\title{
Clinical and Molecular Characteristics Associated with Survival in Advanced Melanoma Treated with Checkpoint Inhibitors
}

\author{
Sunil Badami $\left(\mathbb{D},{ }^{1}\right.$ Sunil Upadhaya $\mathbb{D},{ }^{1}$ \\ Ravi Kanth Velagapudi $\mathbb{D}^{1},{ }^{1}$ Pushyami Mikkilineni $\mathbb{D}^{2},{ }^{2}$ Ranju Kunwor $\mathbb{D}^{\circ},{ }^{3}$ \\ Samer Al Hadidi $\mathbb{D}^{4}{ }^{4}$ and Ghassan Bachuwa $\mathbb{D D}^{1}$ \\ ${ }^{1}$ Hurley Medical Center/Michigan State University, 1 Hurley Plaza, Flint, Michigan 48503, USA \\ ${ }^{2}$ Mercy St. Vincent Medical Center, 2213 Franklin Ave, Toledo, OH 43604, USA \\ ${ }^{3}$ MacNeal Health Center, 3249 South Oak Park Ave. Berwyn, Illinois 60402, USA \\ ${ }^{4}$ Baylor College of Medicine, One Baylor Plaza-BCM 187, Houston, TX 77030, USA \\ Correspondence should be addressed to Sunil Badami; sbadamil@hurleymc.com
}

Received 4 April 2018; Revised 31 May 2018; Accepted 25 June 2018; Published 17 July 2018

Academic Editor: Hailun Wang

Copyright (C) 2018 Sunil Badami et al. This is an open access article distributed under the Creative Commons Attribution License, which permits unrestricted use, distribution, and reproduction in any medium, provided the original work is properly cited.

\begin{abstract}
Background. We performed meta-analysis to gather more evidence regarding clinical-molecular subgroups associated with better overall survival (OS) in advanced melanoma treated with checkpoint inhibitors. Materials and Methods. We performed a systematic search of PubMed, Scopus, Cochrane Library, and clinical trial.gov. Randomized clinical trials that compared a checkpoint inhibitor (nivolumab or pembrolizumab) with investigator choice chemotherapy or ipilimumab were included in our study. Hazard ratios (HR) and confidence interval (CI) were calculated for progression-free survival (PFS) and OS for each subgroup using generic inverse model along with the random effect method. Results. A total of 6 clinical trials were eligible for the meta-analysis. OS was prolonged in wild BRAF subgroup (HR 0.65, 95\% CI 0.49-0.85, p 0.002), Programmed cell death subgroup (PD-1+) (HR 0.57, 95\% CI 0.41-0.80, p 0.001), and high lactate dehydrogenase (LDH) level subgroup (HR 0.60, 95\% CI 0.38-0.95, p 0.03). Similarly, we found increased OS in eastern cooperative oncology group (ECOG) 1 , males and age $>65$ years subgroups. Conclusions. Checkpoint inhibitors significantly increased OS in patients with wild BRAF, positive PD-1, and high LDH. However, results should be interpreted keeping in mind associated significant heterogeneity. The results of this study should help in designing future clinical trials.
\end{abstract}

\section{Introduction}

Advanced melanoma (regionally metastatic melanoma stage III) and metastatic disease (stage IV) has been the deadliest form of cutaneous malignancy. According to the latest statistics from the Surveillance, Epidemiology, and End Results (SEER) 18 registry, the incidence of melanoma in the United States continues to rise. A total of 87,110 cases were reported in 2017. Although there is an uptrend of new cases, the 5-year survival rate has been trending upward, with the latest being $19.9 \%[1]$.

In 2011, a new era began in management of advanced melanoma with United States Food and Drug Administration (FDA) approval of anti-CTLA-4 (cytotoxic T lymphocyte antigen-4) targeted therapy (ipilimumab) [2], which gave promising results, such as better overall survival (OS), response rate, and progression-free survival (PFS).

Other molecular targets were also encouraging, including targeting of B-Rapidly Accelerated Fibrosarcoma (BRAF) gene V600 mutation in 2011[3] (vemurafenib, dabrafenib) and mitogen-activated kinase (MEK) pathway inhibitors (trametinib) approved in 2013[4].

The latest addition to immunotherapy are antiprogramed cell death agents (PD-1), which target the programmed cell death pathway and its ligands. Tumors escape the host immune system by evading checkpoints of $\mathrm{T}$ cells and natural killer cells. To date, the most effective immune checkpoint inhibitor is developed against PD-1 and 
its ligand (PD-L1) [5]. It is also noted that the expression of PD-L1, which is also associated with melanoma, is higher in tumors with poor prognosis $[6,7]$.

The anti-PD-1 agent and monoclonal antibody pembrolizumab got an accelerated approval by the FDA based on the phase 1 study KeyNote (KN) 001 in 2014[8]. It was initially approved for disease progressed on ipilimumab/antiBRAF treatment, but subsequent studies CheckMate (CM) 067, CM 069 (nivolumab), and KN 002 (pembrolizumab) $[9,10]$ proved the superiority of checkpoint inhibitors. As of now, National Comprehensive Cancer Network (NCCN) guidelines recommend these agents either for first-line monotherapy or in combination with CTLA-4 inhibitor.

However, there is not much evidence in terms of which subgroup of patients with advanced melanoma treated with checkpoint inhibitors have better survival outcomes. Available data regarding survival benefit of checkpoint inhibitors in patients based on BRAF status and PD1 expression are contradictory. Results from KN 002 trial and CM 037 trial have shown trend towards better survival in wild BRAF and PD1+ subgroup of patients compared to BRAF mutated and PD1 negative subgroups, respectively, in patients treated with checkpoint inhibitors. However, KN 006 trial, CM 066 trial, and CM 067 trial did not show any survival difference based on BRAF status and PD1 expression [8, 9, 11-13].

As checkpoint inhibitors stimulate immune response of the patient against tumor antigens, response to these drugs is affected by clinical and molecular profile of the patient. Gender, age, and functional status affect immune response [14-16]. Serum lactate dehydrogenase (LDH) is an important staging marker and elevated level is associated with higher tumor burden with worse survival outcomes. Similar to BRAF status and PD1 expression, there is conflicting evidence regarding survival benefit based on $\mathrm{LDH}$ level in patient treated with checkpoint inhibitors $[8,9,11,12]$.

We conducted this meta-analysis and systematic review to gather more evidence regarding survival in different clinical-molecular subgroups based on PD-1 gene expression status, BRAF gene mutation status, serum LDH level, and demographic factors such as age, sex, and ECOG (eastern cooperative oncology group) functional status.

\section{Materials and Methods}

We used the Preferred Reporting Items for Systematic Reviews and Meta-Analyses (PRISMA) model for our analysis (Figure 1) [17]. We performed a systematic search of PubMed, Scopus, Cochrane Library, and clinicaltrial.gov from inception of database till June 2018. Articles published only in English language were considered. We used the following terms in two groups for the search: Group 1: "metastatic melanoma" and "advanced melanoma"; Group 2: "anti-programmed cell death receptor 1 monoclonal antibody", "anti-PD-1 monoclonal antibody", "PD-1", "pembrolizumab" and "nivolumab". We used the all-field strategy (search all text). We used the Boolean operator "OR" for searches using words in the same group. We combined the search results from both groups using the Boolean operator "AND" to narrow down the search. We searched the bibliographies of the retrieved publications to get additional relevant studies. Elaborate search strategy is provided in Supplementary Table 1. For the initial search, we went through the title/abstract of the articles. After the initial search, a full text review of the selected articles was done. We only used published data for the analysis. In addition, we looked for relevant articles in the bibliographies of the selected articles. Initial search resulted in 2641 citations. After exclusion of duplicate citations, abstracts of remaining 1507 articles were reviewed by 2 authors (P.M. and R.K.). Out of 1507 articles 1481 were excluded based on review of abstract and title. Final full text review of remaining 26 articles was done by 3 authors separately (S.B., S.U., and R.V.) and, in case of any conflict during the review, final decision was done by two senior authors (G.B. and S.H.) panel. In case of search yielding more than one paper in the same study population, we chose the most recently published article with outcome relevant to our study.

Inclusion criteria for our analysis followed the PICOS model: population, patients with advanced melanoma; intervention, anti-PD immunotherapy; comparison, anti-PD immunotherapy versus investigator choice chemotherapy or anti CTLA-4 immunotherapy; outcome: clinical outcomes based on clinical and genetic stratification; and study design, randomized studies only [18].

Based on the inclusion criteria, only 6 randomized studies (5, phase III; 1 , phase II) were included in the analysis. Primary outcome and baseline characteristics were extracted by 3 authors (P.M., R.K., and S.B.) (Tables 1, 2, and 3). In case of any confusion, final decision was made by panel of senior authors (G.B. and S.H.). The final Delphi (Table 4) list of included articles was extracted by two authors (S.B. and S.U.). In addition, studies with potentially high risk of bias are listed in Supplementary Table 2.

We used RevMan 5.3 for windows (Cochrane Collaboration, Oxford, United Kingdom) for the analysis. Random models and hazard ratios (HRs) were used to assess the outcomes. PFS and OS were calculated using the generic inverse model along with the random model method. A two-tailed p value of less than 0.05 was taken as significant. Heterogeneity of $<30 \%$ was considered low, 30\%-60\% was considered moderate, and $>60 \%$ was considered substantial.

We conducted meta-regression using Comprehensive Meta-analysis version 3 (Biostat Inc, Englewood, USA). We used categorical (sample size greater or less than 500 and control arm using ipilimumab or not) and continuous (age, gender, ECOG status 0, BRAF mutation, and PD 1 status, year of publication) potential moderator for estimating the effect on heterogeneity and effect size using univariate regression on overall survival rates.

We used funnel plot to assess for publication bias. Funnel plot for this review is symmetrical, so risk of publication bias is low (Supplementary Figure 1).

\section{Results and Discussions}

\subsection{Results}

3.1.1. Overall Survival. Based on BRAF mutation group, we found a significant improvement in the anti-PD-1 group in 


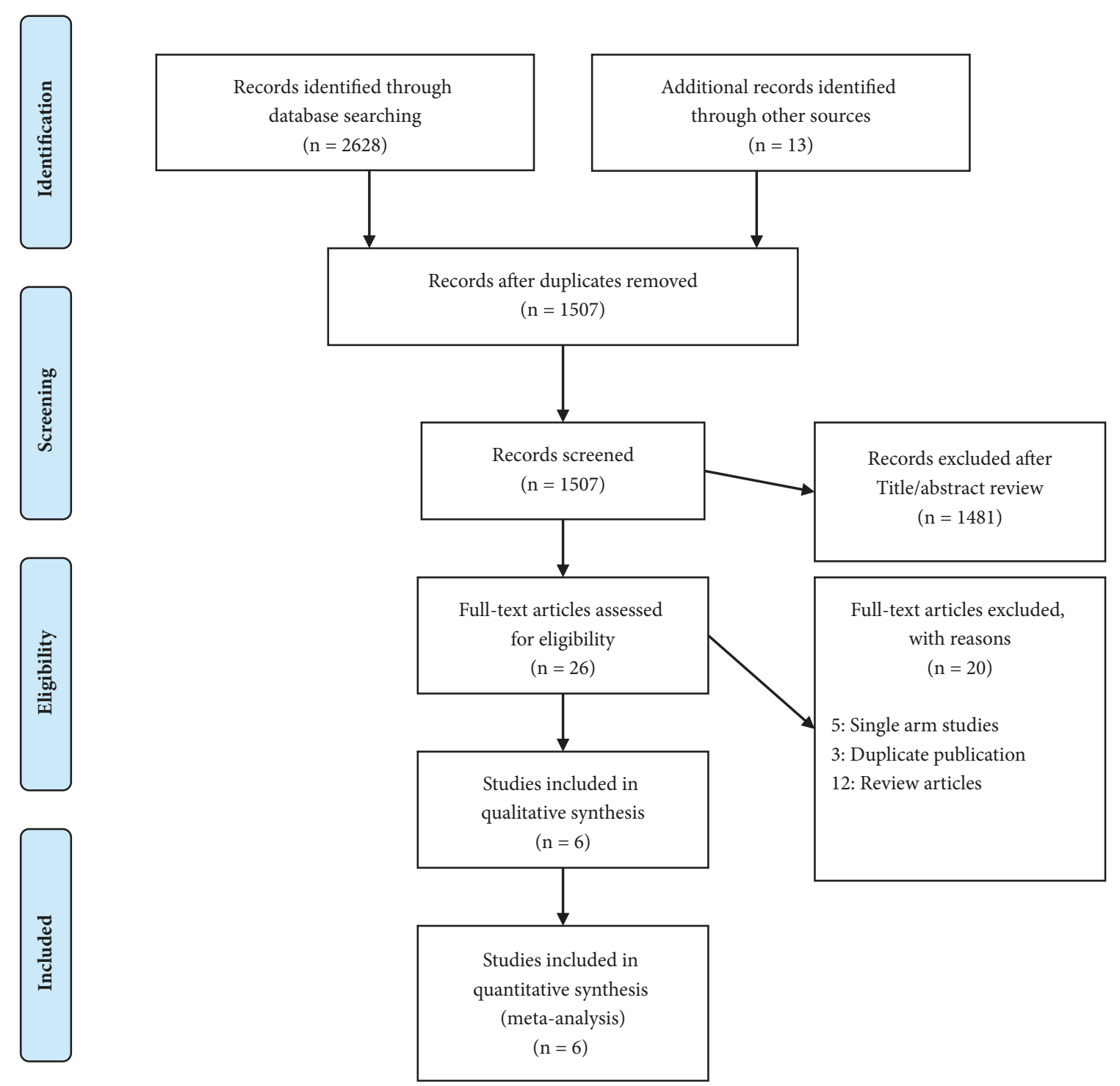

FIgURE 1: PRISMA (Preferred Reporting Items for Systematic Reviews and Meta-Analyses) diagram of the review.

patients with wild BRAF gene (HR 0.65, 95\% CI 0.49-0.85, p value of 0.002 ). This outcome was associated with moderate heterogeneity of $43 \%$ using $\mathrm{I}^{2}$ (Figure 2 ). We did not find a significant difference between the two groups in patients with BRAF mutation (HR 1.10, 95\% CI 0.74-1.62, p value of 0.64 ). Heterogeneity was $0 \%$ for this outcome (Figure 3 ).

Based on PD-1 status, we found a significant improvement in the anti-PD-1 group in patients with PD-1+ (HR $0.57,95 \%$ CI $0.41-0.80$, p value of 0.001 ). This outcome was associated with moderate heterogeneity of $40 \%$ using $\mathrm{I}^{2}$ (Figure 4). We did not find a significant difference between the two groups in patients with PD-1- (HR 0.80, 95\% CI 0.511.27 , p value of 0.35 ).

Heterogeneity was $73 \%$ for this outcome (Figure 5).

Based on ECOG, we found a significant improvement in the anti-PD-1 group in patients with an ECOG of 1 (HR $0.70,95 \%$ CI $0.55-0.90$, $p$ value of 0.004 ). This outcome was associated with very low heterogeneity of $0 \%$. We did not find a significant difference between the two groups in patients with an ECOG of 0 (HR 0.66, 95\% CI 0.41-1.05, p value of $0.08)$. Heterogeneity was $78 \%$ for this outcome.

Based on gender, we found a significant improvement in the anti-PD-1 group in males (HR 0.60, 95\% CI 0.40-0.91, p value of 0.02 ). This outcome was associated with substantial heterogeneity of $73 \%$. We did not find a significant difference between the two groups in females (HR 0.81, 95\% CI 0.61-1.07, $\mathrm{p}$ value of 0.13 ). Heterogeneity was $14 \%$ for this outcome.

Based on age, we found a significant improvement in the anti-PD-1 group in patients age 65 or older (HR 0.61, 95\% CI $0.42-0.88$, $\mathrm{p}$ value of 0.008 ). This outcome was associated with moderate heterogeneity of $41 \%$. We did not find a significant difference between the two groups in patients younger than 65 (HR 0.75, 95\% CI 0.50-1.11, p value of 0.15 ). Heterogeneity was $68 \%$ for this outcome.

Based on lactate dehydrogenase (LDH) levels, we found a significant improvement in the anti-PD-1 group in patients 


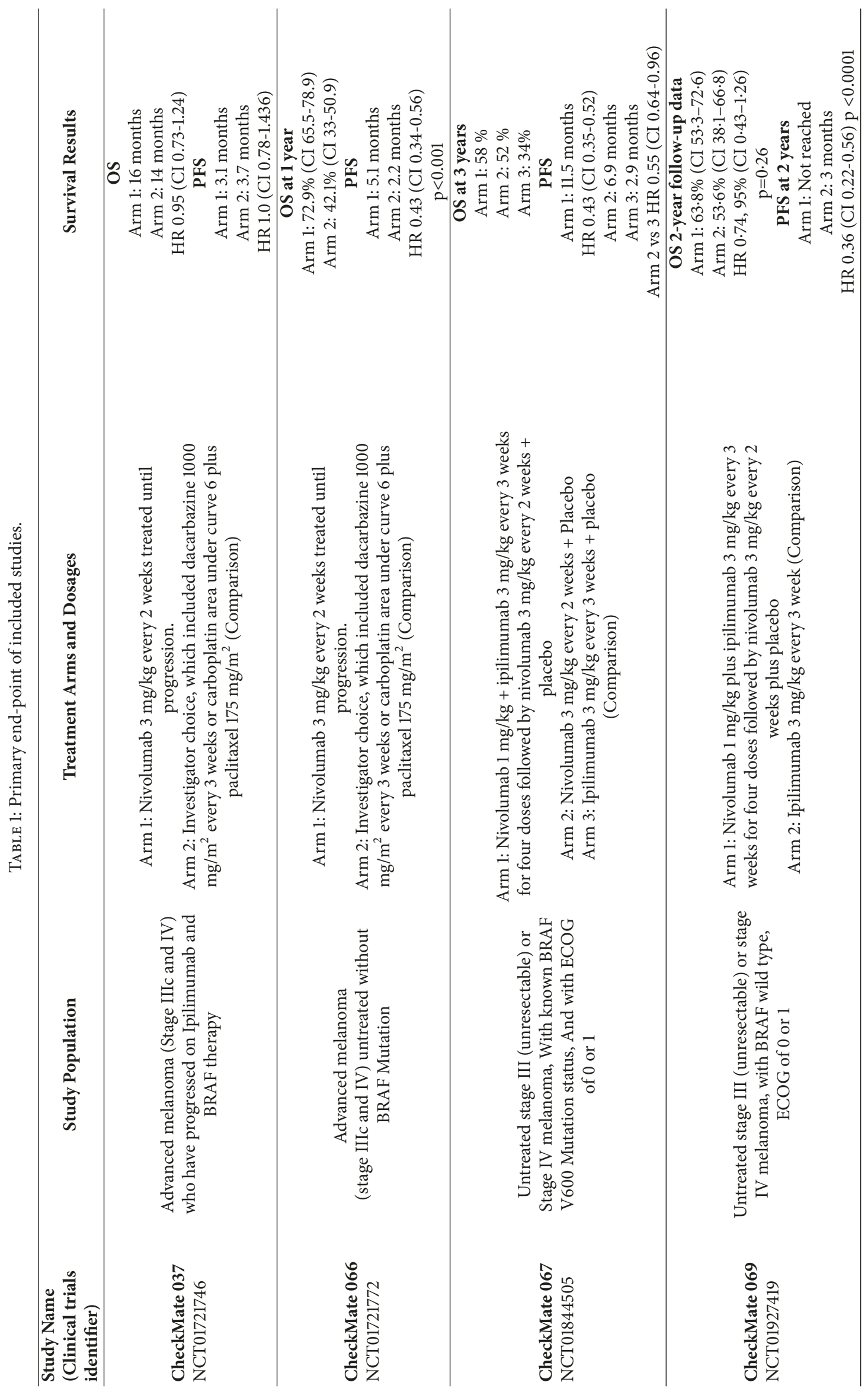




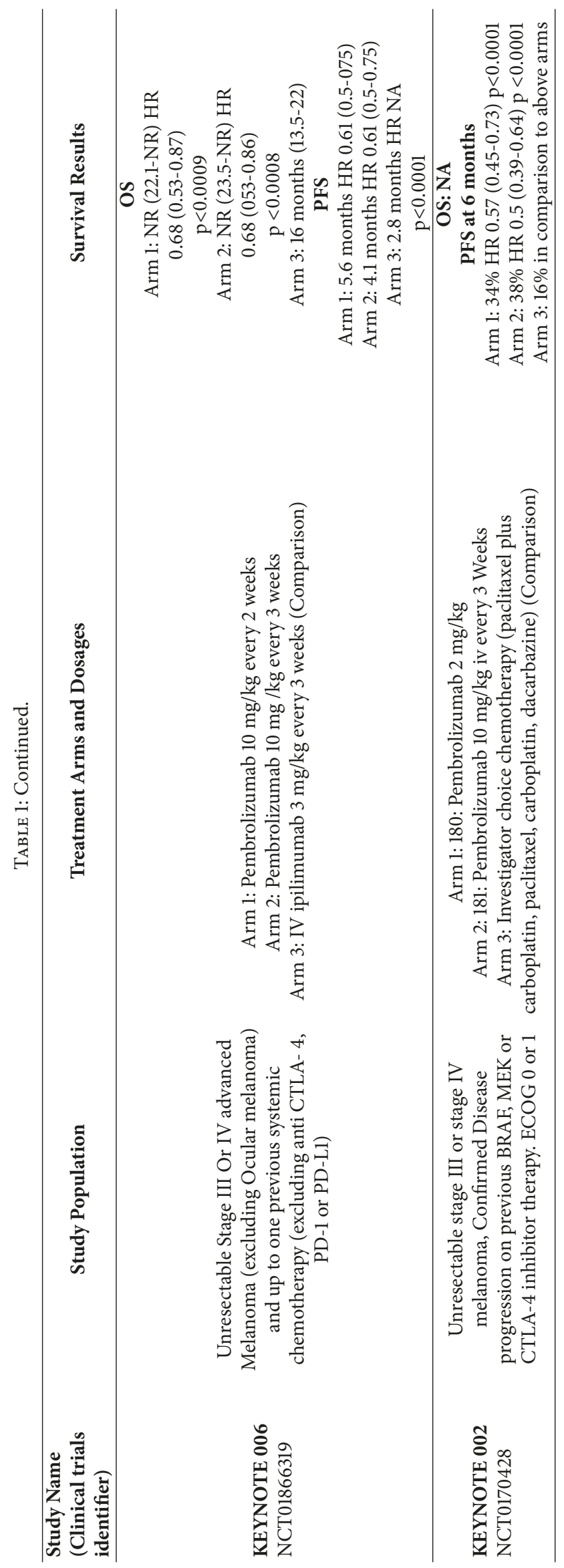




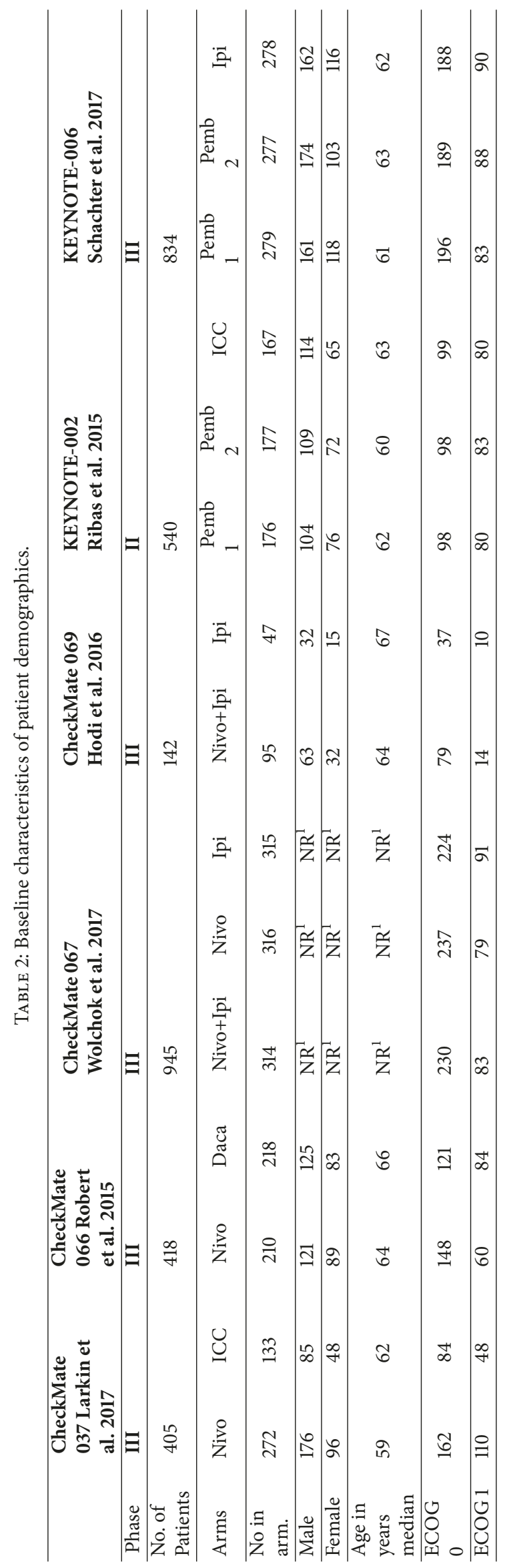




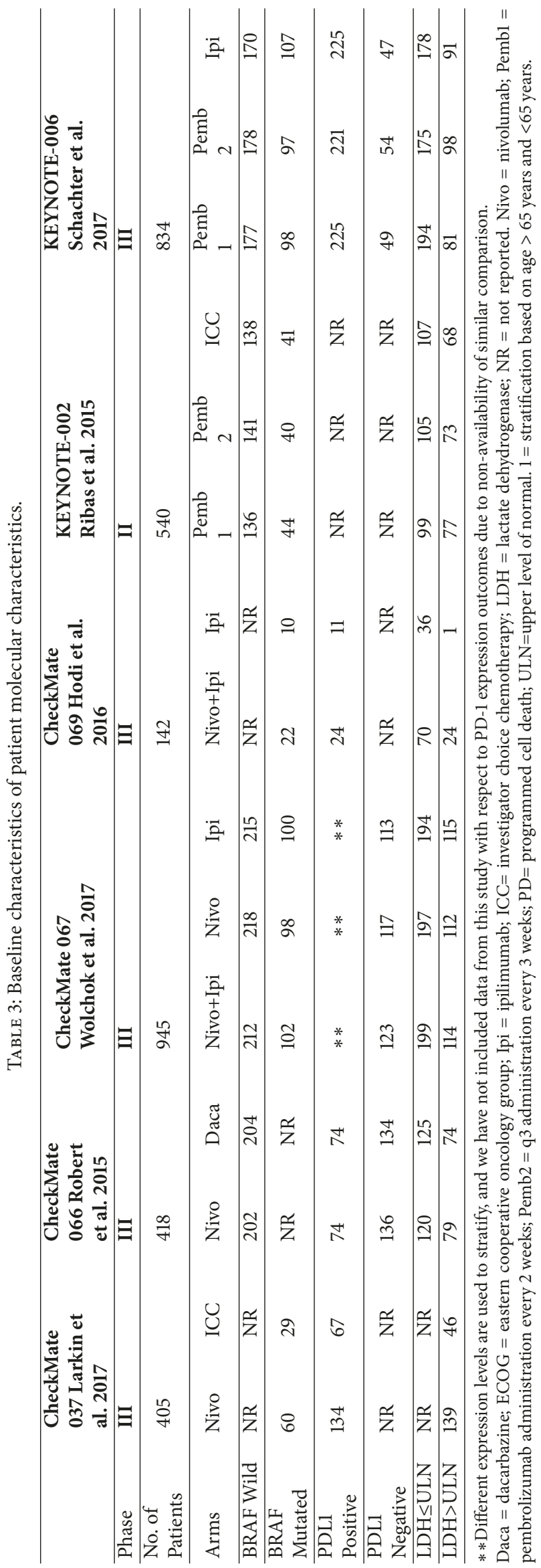




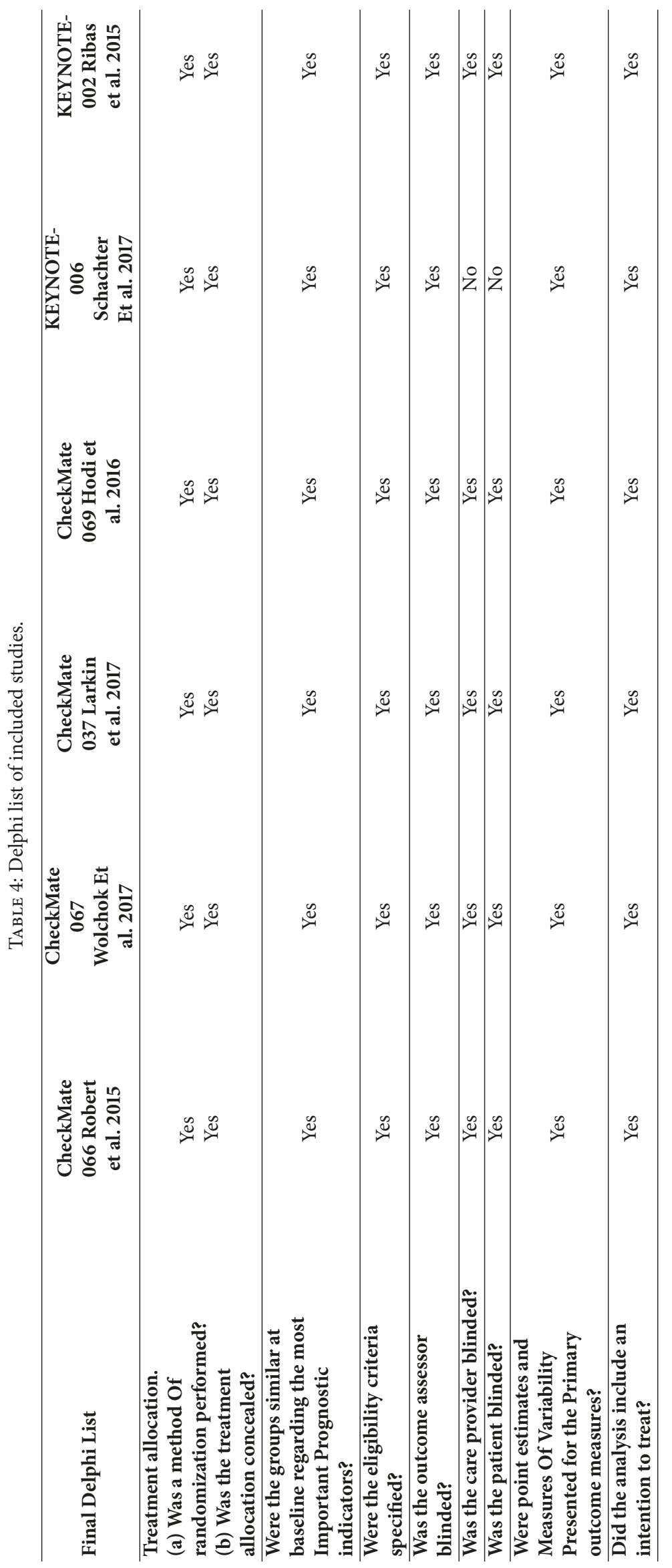




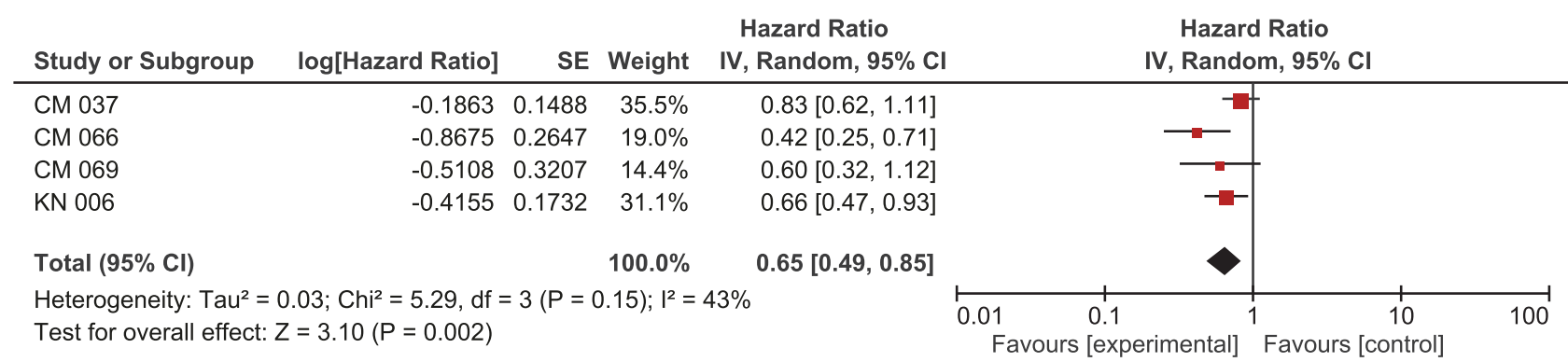

FIGURE 2: Forest plot for overall survival wild BRAF subgroup. CI: confidence interval; CM: CheckMate; KN: KeyNote; IV: inverse variance; SE: standard error.

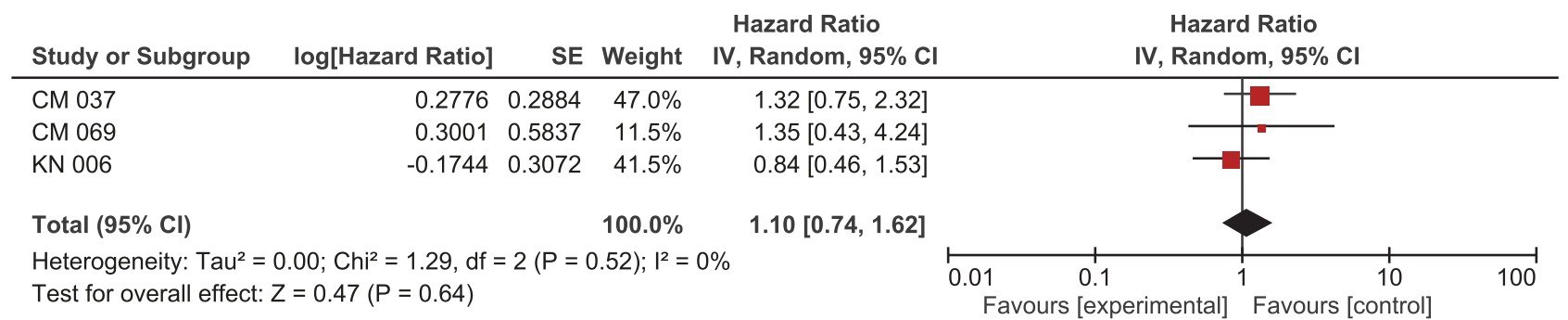

FIGURE 3: Forest plot for overall survival mutated BRAF subgroup. CI: confidence interval; CM: CheckMate; KN: KeyNote; IV: inverse variance; SE: standard error.

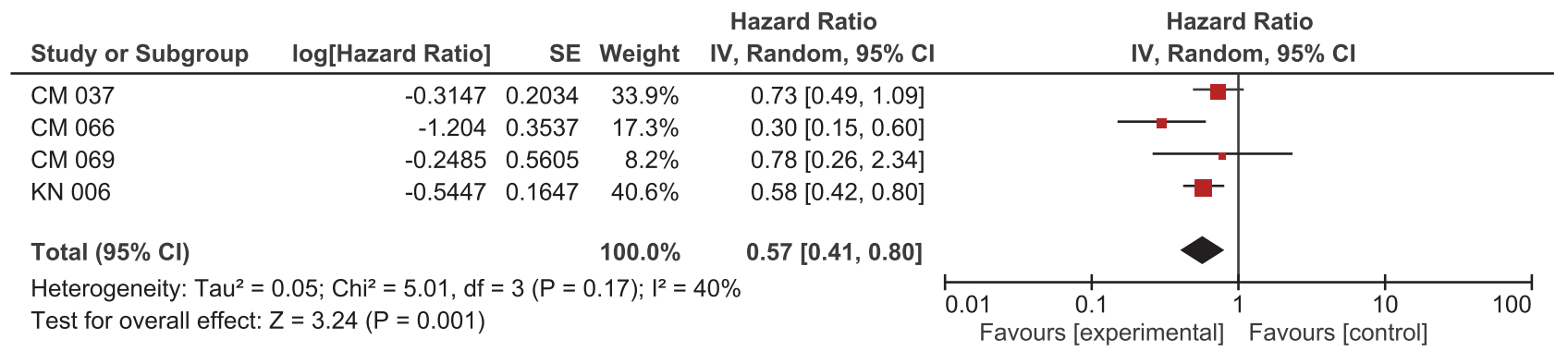

FIGURE 4: Forest plot for overall survival PD1 positive subgroup. CI: confidence interval; CM: CheckMate; KN: KeyNote; IV: inverse variance; SE: standard error.

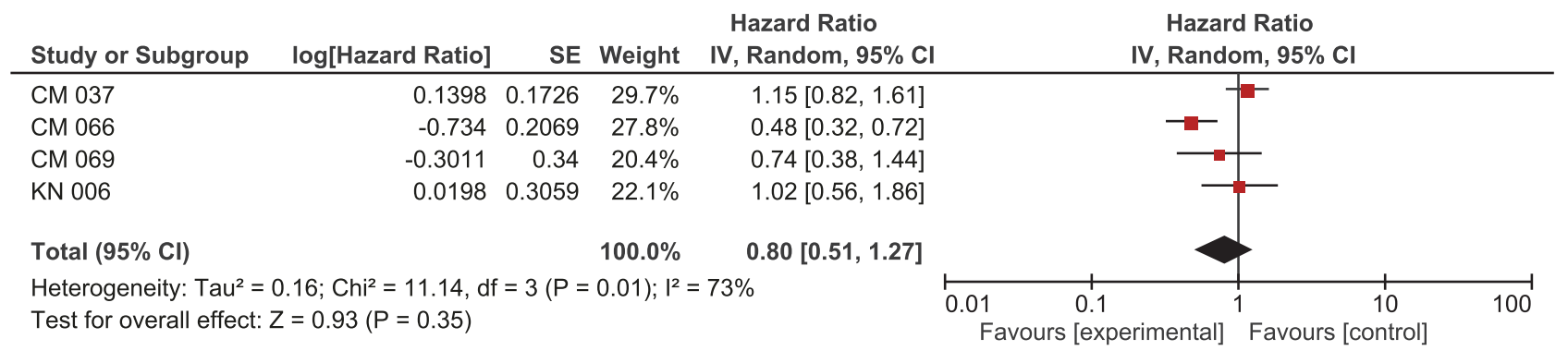

FIGURE 5: Forest plot for overall survival PD1 negative subgroup. CI: confidence interval; CM: CheckMate; KN: Keynote; IV: inverse variance; SE: standard error.

with high LDH levels (HR 0.60, 95\% CI 0.38-0.95, p value of 0.03$)$. This outcome was associated with moderate heterogeneity of 59\% (Figure 6). We did not find a significant difference between the two groups in patients with normal $\mathrm{LDH}$ levels (HR 0.61, 95\% CI 0.36-1.05, p value of 0.07 ). Heterogeneity was $65 \%$ for this outcome (Figure 7).
3.1.2. Progression-Free Survival. Based on BRAF mutation group, we found a significant improvement in the anti-PD1 group in patients with wild BRAF gene (HR 0.49, 95\% CI $0.43-0.55$, $\mathrm{p}$ value of $<0.00001)$. This outcome was associated with very low heterogeneity of $0 \%$ using $\mathrm{I}^{2}$. We did not find a significant difference between the two groups in patients with 


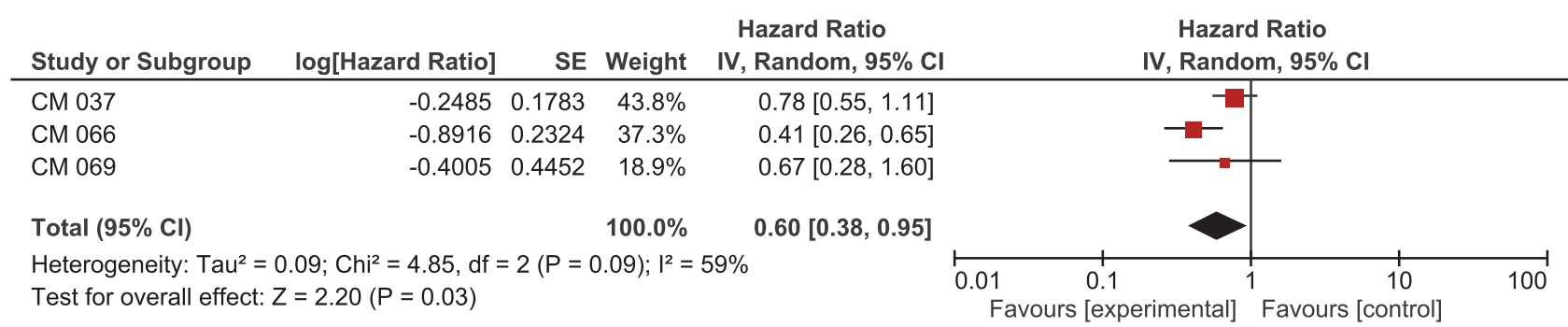

FIGURE 6: Overall survival in high lactate dehydrogenase (LDH) subgroup. CI: confidence interval; CM: CheckMate; KN: KeyNote; IV: inverse variance; SE: standard error.

$\begin{array}{lrrrrr}\text { Study or Subgroup } & \text { log[Hazard Ratio] } & \text { SE } & \text { Weight } & \begin{array}{c}\text { Hazard Ratio } \\ \text { IV, Random, 95\% Cl }\end{array} & \text { Hazard Ratio } \\ \text { IV, Random, 95\% Cl }\end{array}$

FIGURE 7: Overall survival in normal lactate dehydrogenase (LDH) subgroup. CI: confidence interval; CM: CheckMate; KN: KeyNote; IV: inverse variance; SE: standard error.

BRAF mutation (HR 0.73, 95\% CI 0.52-1.04, p value of 0.08 ). Heterogeneity was $26 \%$ for this outcome.

Based on PD-1 status, we found a significant improvement in the anti-PD-1 group in patients with PD-1+ (HR 0.52, 95\% CI $0.40-0.67, \mathrm{p}$ value of $<0.00001)$. This outcome was associated with very low heterogeneity of $0 \%$ using $\mathrm{I}^{2}$. We did not find a significant difference between the two groups in patients with PD-1- (HR 0.48, 95\% CI 0.19-1.20, $\mathrm{p}$ value of 0.12). Heterogeneity was $83 \%$ for this outcome.

Based on ECOG, we found a significant improvement in the anti-PD-1 group in patients with an ECOG of 1 (HR $0.51,95 \%$ CI $0.37-0.69$, p value of $<0.0001)$. This outcome was associated with moderate heterogeneity of $53 \%$. We found a significant difference favoring the anti-PD-1 group in patients with an ECOG of 0 (HR $0.53,95 \%$ CI $0.41-0.68$, p value of $<0.00001)$. Heterogeneity was $0 \%$ for this outcome.

Based on gender, we found a significant improvement in the anti-PD-1 groups in males (HR 0.51, 95\% CI 0.42$0.63, \mathrm{p}$ value of $<0.00001$ ) and females (HR $0.50,95 \% \mathrm{CI}$ $0.36-0.70, \mathrm{p}$ value of $<0.0001)$. This outcome was associated with heterogeneity of $0 \%$ and $36 \%$ in males and females, respectively.

Based on age, we found a significant improvement in the anti-PD-1 groups in patients age 65 or older (HR $0.56,95 \% \mathrm{CI}$ $0.44-0.70, \mathrm{p}$ value of $<0.00001)$ and patients younger than 65 (HR $0.46,95 \%$ CI $0.33-0.65$, p value of $<0.0001$ ). This outcome was associated with heterogeneity of $0 \%$ and $57 \%$ in patients age 65 or older and patients younger than 65, respectively.

Based on lactate dehydrogenase (LDH) levels, We found a significant improvement in the anti-PD-1 groups in patients with high LDH levels (HR 0.59, 95\% CI 0.42-0.83, $\mathrm{p}$ value of 0.003 ) and normal LDH levels (HR 0.41, 95\% CI 0.31-0.53, $\mathrm{p}$ value of $<0.00001)$. This outcome was associated with very low heterogeneity of $0 \%$ in both groups.

Heterogeneity: we did the leave-one-out analysis to find out the cause of high heterogeneity in all outcomes. The cause of high heterogeneity in the outcomes is due to results of CM 066 study. This study only included patients with wild BRAF, so has results strongly favoring PD1 inhibitor compared to other treatment arm.

We also conducted meta-regression to find the cause of heterogeneity. We did not find any impact of age ( $\mathrm{p}$ value 0.26 ), gender ( $p$ value of 0.28 ), PD status ( $p$ value of 0.68 ), year of publication ( $p$ value of 0.42 ), sample size ( $p$ value of 0.65 ), and control arm ( $p$ value of 0.58 ) on heterogeneity. However, ECOG status and LDH levels were associated with decrease in heterogeneity from $46.05 \%$ to $41.41 \%$ ( $\mathrm{p}$ value of 0.28) (Supplementary Figure 2) and $27.29 \%$ (p value of 0.1212 ) (Supplementary Figure 3), respectively; however, both moderators did not reach level of statistical significance. BRAF mutation status was associated with statistically significant decrease in heterogeneity from $46.05 \%$ to $0.00 \%$ (p value of 0.0088) (Supplementary Figure 4).

\section{Discussion}

To the best of our knowledge, this is the first meta-analysis to compare outcomes based on clinical and molecular characteristics of PD-1 antibody use in patients with advanced melanoma. We did not find any significant improvement in OS in patients with BRAF mutation. Similarly, we did not find any improvement in PFS in patients with BRAF mutation. However, both OS and PFS were significantly better in patients with wild-type BRAF. 
To further stratify the results, we did sensitivity analysis, turn by turn leaving out studies using combination therapy of PD-1/CTLA-4 inhibitor and studies using nivolumab. As expected, there was no change in the significance of statistical outcomes in OS and PFS in either of the groups. NCCN guidelines does not have clear recommendation regarding the use of PD-1 inhibitors in advanced melanoma. The NCCN guidelines recommend using BRAF inhibitor in BRAF mutation patients if early response is deemed necessary.

One possible explanation for these results could be due to aggressive nature of melanoma with BRAF mutation compared to wild variant [19]. As of now, evidence for choice of agent in first-line therapy in BRAF mutated patients is indirectly based on retrospective reviews and meta-analysis. However, there is an ongoing clinical trial comparing BRAF/MEK versus checkpoint inhibitors, which hold the key for more concrete evidence.

NCT02224781 is a randomized phase III trial with initial ipilimumab/nivolumab followed by dabrafenib/trametinib at progression versus the reverse approach and the primary outcome for the study is the OS at 2 years of followup. Another trial (NCT02631447) is being conducted in Europe with three treatment groups: initial BRAF/MEK (LGX818/MEK162) followed by ipilimumab/nivolumab at progression, the reverse approach, and the third cohort with 8 weeks of BRAF/MEK with a forced switch to combination immunotherapy, and then BRAF/MEK at progression. These trials are designed to be head-to-head comparisons of efficacy of BRAF/MEK versus CTLA-4/PD-1 therapy; the results will be available no later than 2021 and 2022, respectively.

The PFS was significantly better irrespective of LDH levels in our study compared to investigators choice chemotherapy/ipilimumab. However, we did not find any significant survival benefit in the normal LDH population. We found a significant increase in OS in the PD-1 group with high LDH. In this outcome, a total of only 3 studies were included. After the removal of CM 066[12], which included patients with only wild BRAF, whereas the other 2 studies included patients with wild and mutated BRAF, the statistical significance of the OS in high $\mathrm{LDH}$ patients was lost. In the same CM 066 [12] study, prespecified subgroup analysis OS was significantly improved irrespective of LDH levels. Interestingly, in 2 published studies (one with 78 patients and the other with 617 patients) with mutated BRAF, OS was better in patients with normal LDH than those with high LDH when a BRAF/MEK inhibitor was used $[20,21]$. The association of baseline elevated LDH and poor prognosis is known but utility of LDH as a marker of disease activity and ability to detect occult metastases has been so far ineffective [22]. Although there are three retrospective reviews conducted by Long et al. $[19,23,24]$ which report association of baseline $\mathrm{LDH}$ and serial monitoring of $\mathrm{LDH}$ as an effective marker for targeted therapy, the main drawback remains lack of PDL1 status information, which correlates with better prognosis compared to BRAF mutated patients, as reported in randomized trials and included and confirmed by our results $[9,11]$. In conclusion, evidence regarding association between LDH level and survival outcomes for patients treated with checkpoint inhibitors remains unclear, which needs to be further clarified with future trials with possible monitoring of serum LDH during targeted therapy. As we discussed initially, we did not find a significant benefit in patients with mutated BRAF with the use of a PD-1 inhibitor.

Additionally, there is evidence from a well-sized randomized trial (CM 066) that there was no correlation between $\mathrm{LDH}$ levels and OS [12]. With respect to PD-1 status in our analysis, we found a significant improvement in both OS and PFS in PD-1+ patients. However, the cutoff point for positivity of PD-1 expression in the included studies was not uniform. CM 037, 066, and $069[12,25]$ used 5\% as the cutoff for positivity, whereas $\mathrm{KN} 006$ used $1 \%$ as the cutoff. On sensitivity analysis of OS in patients with positive PD-1 using the leave-one-out method, removal of the only KN006 led to a loss of statistical significance. In CM 037 and CM 069, OS was not statistically significant, irrespective of PD-1 status. However, in CM 066 OS was significant in both groups (PD$1+$ and PD-1 -). One possible hypothesis for these overall results could be that patients with a PD-1 expression between 1 and $5 \%$ behave differently than patients with $<1$ and more than $5 \%$. Further studies should do prespecified subgroup analysis for these kinds of patients.

We found significant OS benefit in subgroups male and age greater than 65 years subgroups compared to female and age less than 65 years, respectively, in patients treated with checkpoint inhibitors. Similar to our results, CM 037 trial showed OS was significantly better for age $>65$-year subgroup compared to $<65$-year subgroup. However, OS in KN 006 trial and CM 066 trial did not correlate with different age. Interestingly, there was significantly better in OS CM 067 trial for age $<65$-year subgroup compared to $>65$-year subgroup. We could not find any possible explanation for this discrepancy. With respect to gender, results of KN 006 were similar to our result. However, OS in CM 037, CM 066, CM067, and CM 069 did not correlate with gender.

Interestingly, we also found significant OS in patients with ECOG 1. However, PFS was significant irrespective of ECOG status, gender, and age. These results can be used to decide treatment of choice and prognostic stratification.

Our meta-analysis has some limitations. First, we did not include individual patient-level data. Second, the results are generalizable only to patient groups eligible for these trials. Significant heterogeneity associated with various outcomes is also an important limitation of our study while interpreting the results. Even though we found significant improvement in overall survival in wild BRAF, PD-1 +, high LDH, Male and age $>65$, these outcomes are associated with heterogeneity of $43 \%, 40 \%, 59 \%, 73 \%$, and $41 \%$, respectively.

Conclusion: Checkpoint inhibitors compared with standard chemotherapy have better survival outcomes in wild BRAF, PD-1 +, high LDH, ECOG 1, Male and age $>65$ years subgroups; however, results are weakened by the significant amount of heterogeneity as discussed above. The outcomes of this analysis will assist in the design of future clinical trials based on prespecified subgroups.

\section{Conflicts of Interest}

The authors report no conflicts of interest. 


\section{Acknowledgments}

This work received funding from Department of Internal Medicine, Hurley Medical Center/Michigan State University, Flint, Michigan, USA. The authors are thankful to Dr. Rodabe N Amaria, M.D., Assistant Professor, Melanoma Division, MD Anderson Cancer Center, Houston, Texas, USA, for reviewing their article and providing them with valuable feedback. They are thankful to Ms. Katherine Negele, Department of Research, Hurley Medical Center/Michigan State Univefvrsity, for English editing of the manuscript.

\section{Supplementary Materials}

Supplementary Figure 1: funnel plot. Supplementary Figure 2: scatter plot of metaregression using ECOG status. Supplementary Figure 3: scatter plot of metaregression using LDH status. Supplementary Figure 4: scatter plot of metaregression using BRAF mutated status. Supplementary Table1: search strategy for meta-analysis. Supplementary Table 2: list of studies with high risk of bias. (Supplementary Materials)

\section{References}

[1] N. Howlader, Am. Noone, M. Krapcho et al., "SEER Data Submission, Posted to the SEER Web Site," SEER Cancer Statistics Review, pp. 1975-2014, April 2017, http://seer.cancer.gov/csr/ 1975_2014/.

[2] F. S. Hodi, S. J. O’Day, D. F. McDermott et al., "Improved survival with ipilimumab in patients with metastatic melanoma," The New England Journal of Medicine, vol. 363, no. 8, pp. 711723, 2010.

[3] P. B. Chapman, A. Hauschild, C. Robert et al., "Improved survival with vemurafenib in melanoma with BRAF V600E mutation," The New England Journal of Medicine, vol. 364, no. 26, pp. 2507-2516, 2011.

[4] A. Hauschild, G. Jean-Jacques, V. Lev Demidov et al., "Dabrafenib in BRAF-Mutated Metastatic Melanoma: A Multicentre, Open-Label, Phase 3 Randomised Controlled Trial," The Lancet, pp. 358-365, 2018.

[5] E. D. Carosella, G. Ploussard, J. LeMaoult, and F. Desgrandchamps, "A Systematic Review of Immunotherapy in Urologic Cancer: Evolving Roles for Targeting of CTLA-4, PD-1/PD-L1, and HLA-G," European Urology, vol. 68, no. 2, pp. 267-279, 2015.

[6] J. Hamanishi, M. Mandai, M. Iwasaki et al., "Programmed cell death 1 ligand 1 and tumor-infiltrating $\mathrm{CD} 8^{+} \mathrm{T}$ lymphocytes are prognostic factors of human ovarian cancer," Proceedings of the National Acadamy of Sciences of the United States of America, vol. 104, no. 9, pp. 3360-3365, 2007.

[7] S. Kraft, M.-T. Fernandez-Figueras, N. A. Richarz, K. T. Flaherty, and M. P. Hoang, "PDL1 expression in desmoplastic melanoma is associated with tumor aggressiveness and progression," Journal of the American Academy of Dermatology, vol. 77, no. 3, pp. 534-542, 2017.

[8] O. Hamid, C. Robert, A. Ribas et al., "Randomized Comparison of Two Doses of the Anti-PD-1 Monoclonal Antibody MK3475 for Ipilimumab-Refractory (IPI-R) and IPI-Naive (IPIN) Melanoma (MEL)," in Journal of Clinical Oncology, ASCO Annual Meeting, 2014.
[9] C. Robert, J. Schachter, G. V. Long et al., "Pembrolizumab versus ipilimumab in advanced melanoma," The New England Journal of Medicine, vol. 372, no. 26, pp. 2521-2532, 2015.

[10] A. Ribas, I. Puzanov, R. Dummer, and et al., "Pembrolizumab versus Investigator-Choice Chemotherapy for IpilimumabRefractory Melanoma (KEYNOTE-002): A Randomised, Controlled, Phase 2 Trial," The Lancet Oncology, vol. 16, no. 8, pp. 908-918, 2015.

[11] J. Larkin, D. Minor, S. D’Angelo et al., "Overall Survival in Patients With Advanced Melanoma Who Received Nivolumab Versus Investigator's Choice Chemotherapy in CheckMate 037: A Randomized, Controlled, Open-Label Phase III Trial," Journal of Clinical Oncology, vol. 36, no. 4, pp. 383-390, 2017.

[12] C. Robert, G. V. Long, B. Brady, C. Dutriaux, and et al., "Nivolumab in previously untreated melanoma without BRAF mutation," The New England Journal of Medicine, vol. 4, pp. 320330, 2015.

[13] J. D. Wolchok, V. Chiarion-Sileni, R. Gonzalez et al., "Overall Survival with Combined Nivolumab and Ipilimumab in Advanced Melanoma," The New England Journal of Medicine, vol. 377, no. 14, pp. 1345-1356, 2017.

[14] C. Wang, B. Dehghani, Y. Li et al., "Membrane estrogen receptor regulates experimental autoimmune encephalomyelitis through up-regulation of programmed death 1," The Journal of Immunology, vol. 182, no. 5, pp. 3294-3303, 2009.

[15] M. J. Polanczyk, C. Hopke, A. A. Vandenbark, and H. Offner, "Estrogen-mediated immunomodulation involves reduced activation of effector $\mathrm{T}$ cells, potentiation of Treg cells, and enhanced expression of the PD-1 costimulatory pathway," Journal of Neuroscience Research, vol. 84, no. 2, pp. 370-378, 2006.

[16] P.-Y. Lin, L. Sun, S. R. Thibodeaux et al., "B7-H1-dependent sex-related differences in tumor immunity and immunotherapy responses," The Journal of Immunology, vol. 185, no. 5, pp. 27472753, 2010.

[17] E. M. Beller, P. P. Glasziou, D. G. Altman et al., "PRISMA for Abstracts: Reporting Systematic Reviews in Journal and Conference Abstracts," PLoS Medicine, vol. 10, no. 4, Article ID e1001419, 2013.

[18] A. M. Methley, S. Campbell, C. Chew-Graham, R. McNally, and S. Cheraghi-Sohi, "PICO, PICOS and SPIDER: A comparison study of specificity and sensitivity in three search tools for qualitative systematic reviews," BMC Health Services Research, vol. 14, no. 1, article no. 579, 2014.

[19] G. V. Long, A. M. Menzies, A. M. Nagrial et al., "Prognostic and clinicopathologic associations of oncogenic BRAF in metastatic melanoma," Journal of Clinical Oncology, vol. 29, no. 10, pp. 1239-1246, 2011.

[20] G. V. Long, J.-J. Grob, P. Nathan et al., "Factors predictive of response, disease progression, and overall survival after dabrafenib and trametinib combination treatment: a pooled analysis of individual patient data from randomised trials," The Lancet Oncology, vol. 17, no. 12, pp. 1743-1754, 2016.

[21] G. V. Long, A. Hauschild, M. Santinami et al., "Adjuvant dabrafenib plus trametinib in stage III BRAF-mutated melanoma," The New England Journal of Medicine, vol. 377, no. 19, pp. 1813-1823, 2017.

[22] D. Kasper, A. Fauci, S. Hauser, and et al., Harrison's Principles of Internal Medicine, Mc GrawHill, 2015.

[23] S. Diem, B. Kasenda, J. Martin-Liberal et al., "Prognostic score for patients with advanced melanoma treated with ipilimumab," European Journal of Cancer, vol. 51, no. 18, pp. 2785-2791, 2015. 
[24] J. Delyon, C. Mateus, D. Lefeuvre et al., "Experience in daily practice with ipilimumab for thetreatment of patients with metastatic melanoma: anearly increase in lymphocyte and eosinophil countsis associated with improved survival," Annals of Oncology, vol. 24, no. 6, pp. 1697-1703, 2013.

[25] M. A. Postow, J. Chesney, A. C. Pavlick et al., "Nivolumab and ipilimumab versus ipilimumab in untreated melanoma," The New England Journal of Medicine, 2015. 


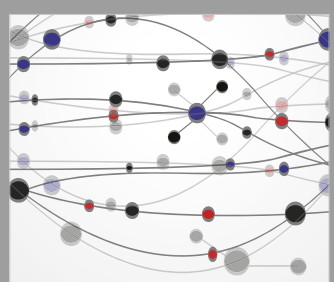

The Scientific World Journal
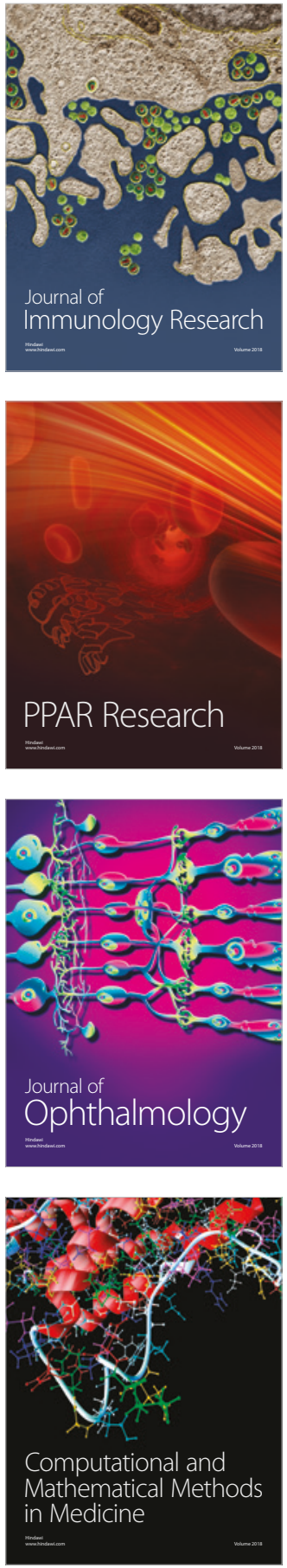

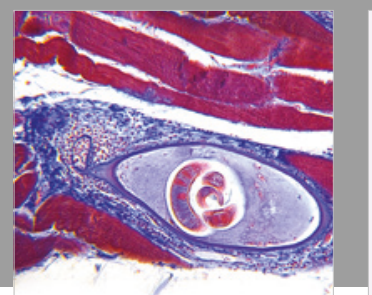

Gastroenterology Research and Practice

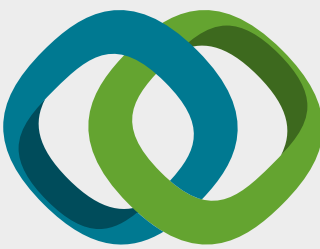

\section{Hindawi}

Submit your manuscripts at

www.hindawi.com
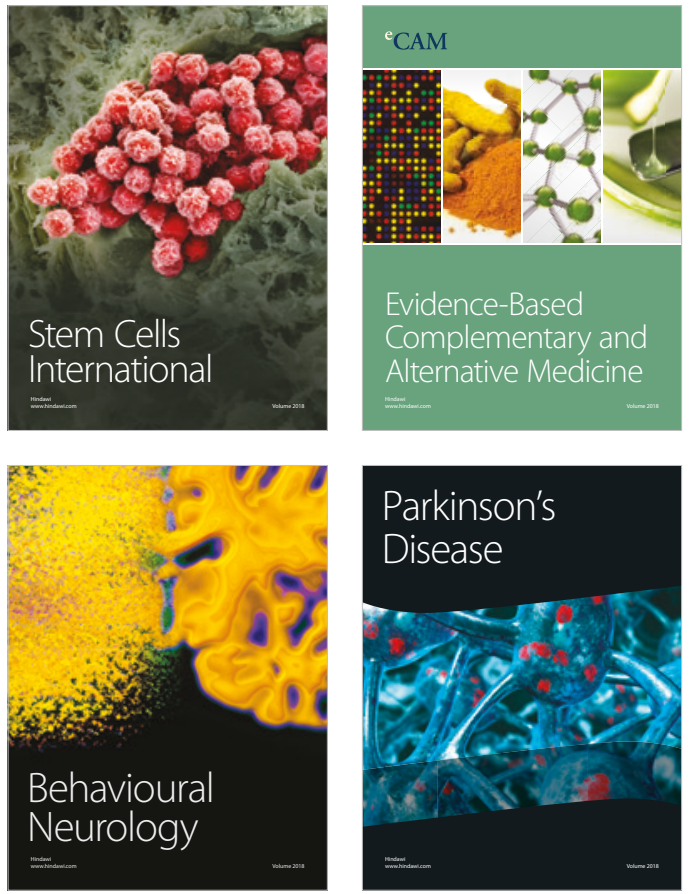

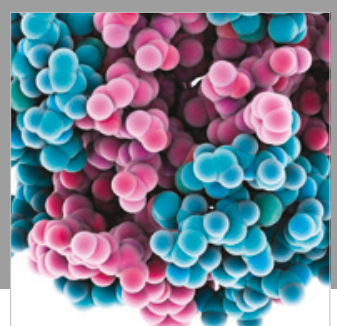

ournal of

Diabetes Research

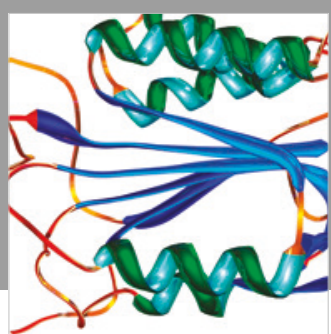

Disease Markers
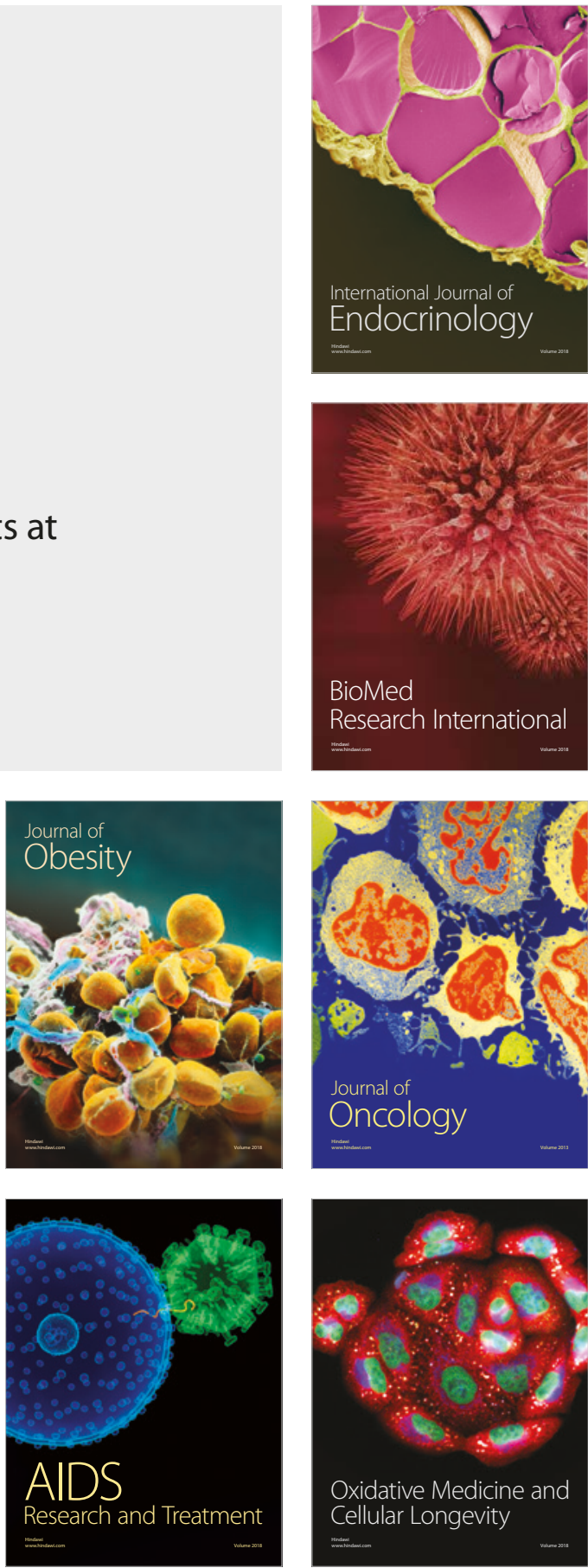